\title{
Prognoseevaluation mittels Immunhistologie
}

\author{
J. Kamarashev \\ G. Burg \\ W. Kempf \\ R. Dummer
}

\section{Immunohistology for Prognostic Evaluation}

\section{Zusammenfassung}

Die Tumordicke ist der wichtigste prognostische Parameter für kutane Melanome. Jedoch wäre es wünschenswert mit zusätzlichen Untersuchungen die Prognose des individuellen Patienten weiter abzusichern. Bewährt haben sich in diesem Zusammenhang in mehreren Studien Proliferationsmarker und auch Adhäsionsmoleküle sowie immunologisch relevante Moleküle wie TAP1, das in der Antigenverarbeitung benötigt wird.

Das maligne Melanom der Haut gehört zu den Tumoren, deren Inzidenz in den letzten Jahrzehnten dramatisch zugenommen hat [1]. Die meisten Patienten mit primären Hautmelanomen werden durch eine Totalexzision mit Sicherheitsabstand adäquat behandelt. Jedoch entwickeln mind. 20\% Metastasen, die dann zum Tod führen. Leider ist es auch heute noch so, dass der Tumor weitgehend therapieresistent wird, nachdem die Metastasierung eingesetzt hat. Die durchschnittliche 5-Jahres-Überlebensrate für Patienten mit primären Melanomen liegt bei etwa $80 \%$ [2]. Nach dem Auftreten von Fernmetastasen wird die Prognose dramatisch verschlechtert und die Patienten haben eine 2-JahresÜberlebensrate von weniger als $10 \%$. Nachdem vermehrt aufwändige adjuvante Therapieverfahren etabliert wurden, müssen jetzt alle Möglichkeiten genützt werden, die dazu beitragen, Hochrisikopatienten zu identifizieren. Eine sichere Identifikation dieser Patientenkollektive würde vielen Patienten unnötige adjuvante Therapien ersparen.

Die Tumordicke und das Stadium der Erkrankung sind die am besten etablierten prognostischen Faktoren $[3,4]$. Weitere prognostisch relevante Aspekte sind Geschlecht, Lokalisation und Ulzeration $[5,6]$.

\section{Abstract}

The tumor thickness is the most important prognostic parameter for cutaneous melanoma. However, additional examinations are desirable to predict the prognosis of the individual patient. In this context, proliferation markers and also adhesion molecules as well as immunological relevant molecules such as TAP1, which is involved in antigen processing, have been shown to be of prognostic relevance.

Immunhistologische Untersuchungen sind von großer Bedeutung bei der Diagnose des Melanoms. Die Rolle dieser Methode bei der Abschätzung der individuellen Prognose weist verschiedene Aspekte auf. Auf der einen Seite sind mehrere immunhistologische Marker mit prognostischer Bedeutung identifiziert worden. Auf der anderen Seite werden immunhistologische Methoden auch zum Nachweis von Mikrometastasen eingesetzt, z.B. im Rahmen der Sentinel-Lymphknotenbiopsie. Daneben ist die Immunhistologie bei der Bestimmung des individuellen Antigen-Profils von Bedeutung. Hierbei kann die Immunhistologie einen prädiktiven Wert haben für einen Vakzinationserfolg, z.B. im Rahmen von Vakzinierungsprotokollen mit Peptiden oder Peptid-gepulsten dendritischen Zellen.

\section{Proliferation und Zellzyklusmarker}

Der Proliferationsmarker Ki-67, erkannt durch den Anti-Ki-67 Antikörper in Gefrierschnitten oder mit dem MIB-1-Antikörper in Paraffin eingebetteten Material ist der beste und auch am genauesten untersuchte Proliferationsmarker. In 4 unabhängigen Studien [7-11] wurde in Multivarianzanalysen nachgewiesen, 
dass er signifikante prognostische Bedeutung hat. Ähnliche Resultate wurden bei Straume et al. [8] in einer Serie von 202 primär kutanen Melanomen in der vertikalen Wachstumsphase und bei Niezabitowski et al. [10] auf der Basis von 93 Läsionen und von Ostmeier et al. [11], die 399 primäre Melanoma untersuchten, dokumentiert. Daneben zeigten Boni et al. [12], dass die Immunreaktivität mit MIB-1 mit dem Metastasierungsverhalten in primären dicken Melanomen, aber nicht in dünnen Melanomen korreliert. Diese Resultate sind auch nachvollziehbar im Rahmen einer Studie von Sparrow et al. [13], der 22 dünne Melanome untersuchte - 11 davon mit Metastasen und 11 ohne. Aufgrund der heute vorliegenden Daten dürfen wir annehmen, dass die Expression von Ki-67 ein wichtiger und von der Tumordicke unabhängiger Parameter für die Prognose beim primären Melanom darstellt, insbesondere für Tumoren mit einer Tumordicke $>1,5 \mathrm{~mm}$.

Daneben gibt es auch Studien, die zeigen, dass Patienten mit einem niedrigen Ki-67-Index in der ersten Metastase eine bessere Prognose aufweisen als Patienten mit einem hohen Index [14], und dass MIB-1-Positivität wiederum ein unabhängiger prognostischer Faktor sowohl für das Gesamtüberleben als auch für das Überleben nach Beginn einer Chemoimmuntherapie für Patienten mit fortgeschrittenem metastasierenden Melanom darstellt [15].

PCNA ist ein anderer Proliferationsmarker, der untersucht wurde. Niezabitowski et al. [10] fanden, dass PCNA ebenfalls ein unabhängiger Prognoseparameter bezüglich „disease-free survival“ und „overall survival“ darstellt.

Veränderungen in der p53-Funktion, einem äußerst wichtigen Tumorsuppressor-Gen, werden als Ursachen für einen Verlust der Zellzykluskontrolle in menschlichen Neoplasien angesehen. Da das gesunde p53-Protein in normalen Zellen sehr schnell abgebaut wird, weist ein Nachweis des p53-Proteins im Zellkern auf ein abnormal stabiles Protein hin, das häufig durch Genmutationen entsteht. Mehrere Studien konnten nachweisen, dass die Überexpression von p53 mit einer ungünstigen Prognose verbunden ist. Yamamoto et al. [18] konnten in einer Studie an 60 primären Melanomen zeigen, dass eine Korrelation zwischen der p53-Überexpression und reduziertem 5-Jahres-Überleben besteht, die durch Untersuchungen von McGregor et al. [19] bestätigt wurde.

Lee et al. [20] untersuchten 21 oberflächlich spreitende und 6 noduläre Melanome und kamen zu dem Ergebnis, dass eine erhöhte p53-Proteinimmunoreaktivität mit einer schlechten Prognose assoziiert ist, ähnlich wie bei Karjalainen et al. [21] im Rahmen einer größeren Studie an 369 Melanompatienten. Andere Studien jedoch konnten diese Ergebnisse nicht nachvollziehen $[10,22,23]$.

Straume et al. [23] untersuchten 102 Patienten mit primär nodulärem Melanom der Haut. In dieser Serie war die Expression von p53 ohne prognostische Bedeutung, jedoch berichteten sie, dass der p16-Verlust signifikant mit deutlich reduziertem rezidivfreien Intervall und Gesamtüberleben assoziiert war. Allerdings nur in der univariablen Analyse. In der multivariablen Analyse war der Verlust von p16 ebenfalls mit häufigen Rezidiven assoziiert. p16 ist ein wichtiges Molekül für die normale Regulation des
Zellzyklus. Sporadische und familiär gehäufte Melanome sind assoziiert mit Mutationen, Verlust von Heterozygotie im p16Lokus. Damit ist p16 sicherlich das am deutlichsten belegte biologisch relevante Tumorsuppressor-Gen beim humanen Melanom.

\section{Marker für melanozytäre Zellen und Bestandteile} des Antigenverarbeitungs-Apparates

S100, HMB45 und Vimentin sind immunhistologische Marker, die regelmäßig für die Diagnose von pigmentierten Hautveränderungen verwendet werden. In vielen Fällen ist ihre diagnostische Bedeutung zweifelsfrei erwiesen, jedoch bleibt ihre Aussagekraft bezüglich prognostischen Fragestellungen unklar. Es gibt jedoch einige Untersuchungen, die darauf hinweisen, dass sie auch prognostische Bedeutung haben $[24,25]$. Eine kürzliche Untersuchung an 93 Fällen stellte diese Frage und kam zu dem Schluss, dass nur HMB45 ein unabhängiger prognostischer Faktor zur Vorhersage des „disease-free survival“ ist [10].

Besonders interessant sind die Melanommarker MAGE-3, Melan A und Tyrosinase, denn Peptide dieser Proteine werden von zytotoxischen T-Lymphozyten erkannt und werden gegenwärtig im Rahmen von Vakzinierungsstudien für Patienten mit metastasierendem Melanom untersucht. Der monoklonale Antikörper 57B entdeckt besonders das MAGE-3-Protein, aber wahrscheinlich auch andere Mitglieder der MAGE-Familie. Färbungen mit diesem Antikörper zeigten eine Spezifität für Melanome von 100\% mit einer Sensitivität von $44 \%$. Über die verschiedenen Stadien gesehen nahm die Positivität von melanozytären Läsionen zu. Bei einem Tumorstadium von T1 zeigten nur $13 \%$ Positivität, bei pT2 22\%, pT3a 29\%, 45\%, pT4 100\%, pTxN1 60\%, und bei Metastasen waren $63 \%$ aller untersuchten Proben positiv [26]. Der monoklonale Antikörper (MAb) A103 weist Melan A/MART1-Protein nach. Mit diesem Antikörper wurden $88 \%$ aller Melanome mit einer sehr hohen Spezifität für melanozytäre Zellen angefärbt. In normaler Haut sind auch normale Melanozyten positiv. Interessanterweise nahm die Anfärbbarkeit über die klinischen Stadien ab. Während im Stadium I praktisch alle untersuchten Fälle positiv waren, waren es im Stadium II nur noch $88 \%$, im Stadium III 90\% und im Stadium IV 75\% [27]. Der Antikörper T311 färbt das Tyrosinase-Protein im histologischen Schnitt an. Eine Färbung mit diesem Antikörper war positiv bei 94\% aller Melanome mit einer sehr hohen Spezifität für melanozytäre Zellelemente. Wiederum fand sich eine negative Korrelation zwischen Immunpositivität und klinischem Stadium. Frühstadien (Stadium I und II) waren immer positiv, im Stadium III und IV waren noch $86 \%$ der Fälle positiv [28]. Diese Färbungen sind von besonderer Bedeutung für Patienten, die im Rahmen von spezifischen Immuntherapien wie Vakzinierung behandelt werden sollen, da die Expression dieser Tumorantigene dazu beitragen könnte, die Chancen für eine erfolgreiche Therapie abzuschätzen. Es ist auch gezeigt worden, dass die gleichzeitige Downregulation von Melan-A und HLA A2 bei Melanomzellen mit einer negativen Prognose verbunden ist [29]. Eine andere Studie hat darauf hingewiesen, dass die Expression von MAGE-3 positive prognostische Bedeutung haben könnte [30]. Allerdings müssen diese Untersuchungsergebnisse im Rahmen größerer Studien, die sich speziell mit der prognostischen Bedeutung dieser Marker beschäftigen, untersucht werden. 
Wir wissen heute, dass das Melanom ein antigener Tumor ist, der Immunantworten erzeugen kann. Eine effektive Präsentation von melanozytären oder tumorassoziierten Antigenen ist wichtig für die Induktion dieser Immunantwort. Defekte im Antigenverarbeitungsapparat können somit in Immune Escape-Phänomene münden und zum Fortschreiten der Erkrankung beitragen. Wir haben die Expression verschiedener Komponenten des Antigenverarbeitungsapparates in primären Melanomen untersucht und diese Ergebnisse mit der Tumordicke und dem Verlauf korreliert. Dabei wurden 17 primäre Melanome mit einer Tumordicke unter $0,7 \mathrm{~mm}$ und 21 mit einer Tumordicke über $1,5 \mathrm{~mm}$ mit verschiedenen Antikörpern gefärbt (anti-LMP2, -LMP7, -TAP1, -TAP2, -HLA Klasse I und II). 20 Patienten entwickelten keine Metastasen in der Nachbeobachtungszeit, die durchschnittlich 10,5 Jahre betrug. 18 Patienten rezidivierten innerhalb durchschnittlich 15 Monaten nach der Exzision. Sehr häufig fanden wir vermindert exprimierte Marker. Häufig waren die LMP- und TAPUntereinheiten vermindert exprimiert (40\%) und fast immer HLA-Klasse-I-Moleküle (ca. 70\%). Die Expression dieser Marker war nicht mit der Tumordicke korreliert. Nur die Expression TAP1 und TAP2 war signifikant korreliert mit dem Risiko, Metastasen zu entwickeln. Diese Korrelation war unabhängig von der Tumordicke für TAP1. Aufgrund dieser Daten gehen wir davon aus, dass TAP1- und möglicherweise auch TAP2-Expression in primären Melanomen einen unabhängigen prognostischen Marker darstellt. Abnormalitäten in der Antigenpräsentation können erklären, warum keine absolute Korrelation zwischen Tumordicke und Prognose besteht [31].

In früheren Arbeiten war auch die HLA-Klasse-II-Expression als prognostischer Parameter beschrieben worden. Ostmeier et al. [32] berichteten, dass die HLA-DR-Expression ein ungünstiger prognostischer Marker ist. In einer neueren Studie wurde diese bestätigt. Diese negative prognostische Bedeutung erscheint paradox, da diese Moleküle durch Interferon $\gamma$ hochreguliert werden können und dann eine verbesserte Antigenpräsentation erfolgen könnte. Jedoch ist eine erhöhte HLA-Klasse-II-Expression nicht mit einer verbesserten T-Zellstimulation assoziiert, was erklärbar wird durch das Fehlen von kostimulatorischen Molekülen [33].

\section{Adhäsionsmoleküle und Zellmatrix-Interaktionen}

Tumorwachstum und Metastasierung beinhalten eine komplexe Kaskade koordinierter Zellfunktionen. Wichtige beteiligte Untereinheiten sind in diesem Zusammenhang Zelladhäsionsmoleküle. Integrine, Katherine und Adhäsionsmoleküle der Immunglobulin-Superfamilien sind in der Tumorprogression und Metastasierung des kutanen Melanoms beteiligt. Die Expression des $\alpha$ vs. $\beta$-3-Integrins ist ein früher Schritt beim Wechsel des Wachstumsverhaltens von der radialen zur vertikalen Wachstumsphase. Ist dieses Molekül in primären Hautläsionen vorhanden, besteht ein erhöhtes Rezidivrisiko [34]. Die Expression von VLA-4 ist charakteristisch für fortgeschrittene primäre Melanome und könnte in der Interaktion von Tumorzellen mit VCAM-1 auf Endothelien beteiligt sein. Auch für die Expression von VLA-4 in Primärläsionen gilt, dass es negative prognostische Bedeutung aufweist [35]. In dieser Studie wurde auch für die Expression von ELAM-1 (E-Selektin) und CD62 (P-Selektin) auf intratumoralen Gefäßen eine prognostische Bedeutung nachgewiesen.
Das Adhäsionsmolekül ICAM-1 gehört zur Familie der Immunglobulin-Superfamilie. ICAM-1-Expression auf primären Melanomen korreliert signifikant mit der Tumordicke und einem ungünstigen Verlauf [36]. Möglicherweise sind in diesem Zusammenhang lösliche ICAM-1-Moleküle für diesen negativen Effekt verantwortlich [37]. In einer anderen Arbeit wurde darauf hingewiesen, dass die gleichzeitige Expression von ICAM-1 und GMGSF beim metastasierenden Melanom signifikant mit einem kürzeren rezidivfreien Intervall verknüpft ist [38].

Proteasen sind wichtige Moleküle für die Invasion, die Ausbreitung und die Metastasierung verschiedener Tumoren. Auch hier ist eine ganze Reihe von Molekülen wie Kollagenase, Plasminogen-Aktivatoren, Stromelysin und andere beteiligt. So wurde z.B. CatB als wichtiger Faktor für die Metastasierung nachgewiesen. CatD könnte in der frühen Phase (Entwicklung von dysplastischen Naevi zum Melanom) eine Rolle spielen [39]. Dickere primäre Tumoren zeigen häufig erhöhte Expression von CatD und CatB und auch Kollagenase IV, falls ein hohes Metastasierungsrisiko besteht [40].

Die Überexpression der 72-kD-Typ-IV-Kollagenase in primären Melanomen ist ein unabhängiger prognostischer Faktor für das gesamte Überleben von Melanompatienten [41].

Osteonektin ist ein weiteres Glykoprotein, das in verschiedenen Zellmatrix-Interaktionen und in der Angiogenese beteiligt ist. Die Expression von Osteonektin in dünnen Primärmelanomen ist signifikant korreliert mit einem Progressions- $(\mathrm{p}=0,01)$ und Metastasierungsrisiko $(p=0,005)$ und im Gesamtüberleben $(p=0,03)[42]$.

\section{Schllussfolgerung}

Mit der Zunahme der Melanomfälle gewinnt das Problem der prognostischen Zuordnung und daran angepasst die Auswahl der Therapie für den einzelnen Patienten immer größere Bedeutung. Dieses Problem wurde von verschiedenen Arbeitsgruppen

Tab. 1 Prognostische Marker und ihre biologische Relevanz

\begin{tabular}{|ll}
\hline prognostische Marker & biologische Bedeutung \\
\hline Ki-67 & $\begin{array}{l}\text { Proliferationsmarker (exprimiert in späten } \mathrm{G}_{1} \text {, } \\
\text { S-, } \text { M-, } \mathrm{G}_{2} \text {-Phasen des Zellzyklus, aber nicht in } \mathrm{G}_{0} \text { ) }\end{array}$ \\
\hline PCNA & $\begin{array}{l}\text { Proliferationsmarker } \\
\text { (exprimiert in der S-Phase des Zellzyklus) }\end{array}$ \\
\hline p53 & Tumorsuppressorgen \\
\hline P16 & Zellzyklusregulator \\
\hline HMB45 & melanozytärer Differenzierungsmarker (gP 100) \\
\hline MAGE3 & Cancer-Testis-Antigen \\
\hline Melan A/MART-1 & melanozytärer Differenzierungsmarker \\
\hline TAP1 & Antigenverarbeitung (Transportermolekül) \\
\hline TAP2 & Antigenverarbeitung (Transportermolekül) \\
\hline LMP & Antigenverarbeitung \\
\hline HLA Klasse I und II & Antigenpräsentation \\
\hline$\alpha-$ vs. $\beta$-3-Integrin & Zelladhäsion \\
\hline VLA-4 & Adhäsion des vaskulären Endotheliums \\
\hline ICAM-1 & Zelladhäsion \\
\hline Cathepsine & extrazelluläre Matrix-Interaktionen \\
\hline Collagenase IV & Protease \\
\hline
\end{tabular}


angegangen. Die Analyse neuer immunhistologischer Marker ist sicherlich ein wichtiger Beitrag in diesem Zusammenhang. Die oben dargestellten Fortschritte können jedoch eine rationale Basis für die Identifizierung von Hochrisikopatienten in der $\mathrm{Zu}$ kunft bieten. Jedoch müssen die jetzt vorhandenen prognostischen Marker in größeren Multizenterstudien in ihrer prognostischen Bedeutung überprüft werden, so dass langfristig nur wenige immunhistologische Färbungen für diese Fragestellung eingesetzt werden müssen (Tab.1).

\section{Literatur}

${ }^{1}$ Berwick M, Halpern A. Melanoma epidemiology. Curr Opin Oncol 1997; 9: 178-182

${ }^{2}$ Häffner AC, Garbe C, Burg G, Buttner P, Orfanos CE, Rassner G. The prognosis of primary and metastasising melanoma. An evaluation of the TNM classification in 2,495 patients. Br J Cancer 1992; 66: $856-861$

${ }^{3}$ Dummer R, Bösch U, Panizzon R, Bloch PH, Burg G für die Fachkommission „Hautkrebs“. Swiss Guidelines for the treatment and followup of cutaneous melanoma. Dermatology 2001; 203: 75-80

${ }^{4}$ Thorn M, Ponten F, Bergstrom R, Sparen P, Adami HO. Clinical and histopathologic predictors of survival in patients with malignant melanoma: a population-based study in Sweden. J Natl Cancer Inst 1994; 86: $761-769$

${ }^{5}$ Karakousis CP, Driscoll DL. Prognostic parameters in localised melanoma: gender versus anatomical location. Eur J Cancer 1995; 3: $320-324$

${ }^{6}$ Garbe C, Buttner P, Bertz J, Burg G, d'Hoedt B, Drepper H et al. Primary cutaneous melanoma. Prognostic classification of anatomic location. Cancer 1995; 75: $2492-2498$

${ }^{7}$ Ramsay JA, From L, Iscoe NA, Kahn HJ. MIB-1 proliferative activity is a significant prognostic factor in primary thick cutaneous melanomas. J Invest Dermatol 1995; 105: 22 - 26

${ }^{8}$ Straume O, Sviland L, Akslen LA. Loss of nuclear p16 protein expression correlates with increased tumor cell proliferation (Ki-67) and poor prognosis in patients with vertical growth phase melanoma. Clin Cancer Res 2000; 6: 1845-1853

${ }_{9}^{9}$ Korabiowska M, Brinck U, Middel P, Brinkmann U, Berger H, Radzun HJ et al. Proliferative activity in the progression of pigmented skin lesions, diagnostic and prognostic significance. Anticancer Res 2000; 20: $1781-1785$

${ }^{10}$ Niezabitowski A, Czajecki K, Rys J, Kruczak A, Gruchala A, Wasilewska A et al. Prognostic evaluation of cutaneous malignant melanoma: a clinicopathologic and immunohistochemical study. J Surg Oncol 1999; 70: $150-160$

${ }^{11}$ Ostmeier H, Fuchs B, Otto F, Mawick R, Lippold A, Krieg V et al. Prognostic immunohistochemical markers of primary human melanomas. Br J Dermatol 2001; 145: $203-209$

12 Boni R, Doguoglu A, Burg G, Muller B, Dummer R. MIB-1 immunoreactivity correlates with metastatic dissemination in primary thick cutaneous melanoma. J Am Acad Dermatol 1996; 35: 416-418

13 Sparrow LE, English DR, Taran JM, Heenan PJ. Prognostic significance of MIB-1 proliferative activity in thin melanomas and immunohistochemical analysis of MIB-1 proliferative activity in melanocytic tumors. Am J Dermatopathol 1998; 20: 12 - 16

${ }^{14}$ Hernberg M, Turunen JP, von Boguslawsky K, Muhonen T, Pyrhonen S. Prognostic value of biomarkers in malignant melanoma. Melanoma Res 1998; 8: 283-291

15 Vlaykova T, Talve L, Hahka-Kemppinen M, Hernberg M, Muhonen T, Franssila K et al. MIB-1 immunoreactivity correlates with blood vessel density and survival in disseminated malignant melanoma. Oncology 1999; 57: $242-252$

${ }^{16}$ Gelsleichter L, Gown AM, Zarbo RJ, Wang E, Coltrera MD. p53 and mdm-2 expression in malignant melanoma: an immunocytochemical study of expression of $\mathrm{p} 53, \mathrm{mdm}-2$, and markers of cell proliferation in primary versus metastatic tumors. Mod Pathol 1995; 8: 530-535

17 Loggini B, Rinaldi I, Pingitore R, Cristofani R, Castagna M, Barachini P. Immunohistochemical study of 48 cutaneous melanomas: p53, PCNA Bcl-2 expression and multidrug resistance. Tumori 2001; 87: 179- 186
${ }^{18}$ Yamamoto M, Takahashi H, Saitoh K, Horikoshi T, Takahashi M. Expression of the $\mathrm{p} 53$ protein in malignant melanomas as a prognostic indicator. Arch Dermatol Res 1995; 287: 146-151

${ }^{19}$ McGregor JM, Yu CC, Dublin EA, Barnes DM, Levison DA, MacDonald DM. p53 immunoreactivity in human malignant melanoma and dysplastic naevi. Br J Dermatol 1993; 128: 606-611

20 Lee CS, Pirdas A, Lee MW. p53 in cutaneous melanoma: immunoreactivity and correlation with prognosis. Australas J Dermatol 1995; 36: $192-195$

${ }^{21}$ Karjalainen JM, Eskelinen MJ, Kellokoski JK, Reinikainen M, Alhava EM, Kosma VM. p21 (WAF1/CIP1) expression in stage I cutaneous malignant melanoma: its relationship with p53, cell proliferation and survival. Br J Cancer 1999; 79: 895-902

22 Weiss J, Heine M, Korner B, Pilch H, Jung EG. Expression of p53 protein in malignant melanoma: clinicopathological and prognostic implications. Br J Dermatol 1995; 133: 23 - 31

${ }^{23}$ Straume O, Akslen LA. Alterations and prognostic significance of p16 and p53 protein expression in subgroups of cutaneous melanoma. Int J Cancer 1997; 74: 535-539

${ }^{24}$ Fernando SS, Johnson S, Bate J. Immunohistochemical analysis of cutaneous malignant melanoma: comparison of S-100 protein, HMB-45 monoclonal antibody and NKI/C3 monoclonal antibody. Pathology 1994; 26: $16-19$

${ }^{25}$ Mirecka J, Korabiowska M, Schauer A. Comparative distribution of S-100 protein and antigen HMB-45 in various types of melanomas and naevi. Pol J Pathol 1995; 46: 167-172

${ }^{26}$ Hofbauer GF, Schaefer C, Noppen C, Boni R, Kamarashev J, Nestle FO et al. MAGE-3 immunoreactivity in formalin-fixed, paraffin-embedded primary and metastatic melanoma: frequency and distribution. Am J Pathol 1997; 151: 1549-1553

${ }^{27}$ Hofbauer GF, Kamarashev J, Geertsen R, Boni R, Dummer R. Melan A/MART-1 immunoreactivity in formalin-fixed paraffin-embedded primary and metastatic melanoma: frequency and distribution. Melanoma Res 1998; 8: 337-343

${ }^{28}$ Hofbauer GF, Kamarashev J, Geertsen R, Boni R, Dummer R. Tyrosinase immunoreactivity in formalin-fixed, paraffin-embedded primary and metastatic melanoma: frequency and distribution. J Cutan Pathol 1998; 25: 204-209

${ }^{29}$ Kageshita T, Kawakami Y, Ono T. Clinical significance of MART-1 and HLA-A2 expression and CD8+ T cell infiltration in melanocytic lesions in HLA-A2 phenotype patients. J Dermatol Sci 2001; 25: 36 - 44

${ }^{30}$ Busam KJ, Iversen K, Berwick M, Spagnoli GC, Old LJ, Jungbluth AA. Immunoreactivity with the anti-MAGE antibody 57B in malignant melanoma: frequency of expression and correlation with prognostic parameters. Med Pathol 2000; 13: 459-465

${ }^{31}$ Kamarashev J, Ferrone S, Seifert B, Boni R, Nestle FO, Burg G et al. TAPI down-regulation in primary melanoma lesions: an independent marker of poor prognosis. Int J Cancer 2001; 95: 23-28

32 Ostmeier H, Fuchs B, Otto F, Mawick R, Lippold A, Krieg V et al. Can immunohistochemical markers and mitotic rate improve prognostic precision in patients with primary melanoma? Cancer 1999; 85: $2391-2399$

33 Townsend SE, Allison JP. Tumor rejection after direct costimulation of CD8+ T cells by B7-transfected melanoma cells. Science 1993; 259: $368-370$

${ }^{34}$ Natali PG, Hamby CV, Felding-Habermann B, Liang B, Nicotra MR, DiFilippo $\mathrm{F}$ et al. Clinical significance of alpha(v)beta 3 integrin and intercellular adhesion molecule- 1 expression in cutaneous malignant melanoma lesions. Cancer Res 1997; 57: 1554-1560

${ }^{35}$ Schadendorf D, Heidel J, Gawlik C, Suter L, Czarnetzki BM. Association with clinical outcome of expression of VLA-4 in primary cutaneous malignant melanoma as well as P-selectin and E-selectin on intratumoral vessels. J Natl Cancer Inst 1995; 87: 366 - 371

${ }^{36}$ Natali P, Nicotra MR, Cavaliere R, Bigotti A, Romano G, Temponi M et al. Differential expression of intercellular adhesion molecule 1 in primary and metastatic melanoma lesions. Cancer Res 1990; 50: $1271-1278$

37 Becker JC, Dummer R, Burg G, Schmidt RE. Shedding of ICAM-1 from human melanoma cell lines induced by IFN-gamma and TNF-alpha: Functional consequences on cell mediated cytotoxicity. J Immunol 1991; 147: 4398 - 4401

38 Ciotti P, Pesce GP, Cafiero F, Rainero ML, Sementa A, Nicolo G et al. Intercellular adhesion molecule-1 (ICAM-1) and granulocyte-macrophage colony stimulating factor (GM-CSF) co-expression in cutaneous malignant melanoma lesions. Melanoma Res 1999; 9: 253-260 
${ }^{39}$ Podhajcer OL, Bover L, Bravo AI, Ledda MF, Kairiyama C, Calb I et al. Expression of cathepsin D in primary and metastatic human melanoma and dysplastic nevi. J Invest Dermatol 1995; 104: 340 - 344

${ }^{40}$ Goldmann T, Ribbert D, Suter L, Brode M, Otto F. Tumor characteristics involved in the metastatic behaviour as an improvement in primary cutaneous melanoma prognostics. J Exp Clin Cancer Res 1998; 17: $483-489$
${ }^{41}$ Vaisanen A, Kallioinen M, Taskinen PJ, Turpeenniemi-Hujanen T. Prognostic value of MMP-2 immunoreactive protein (72kD type IV collagenase) in primary skin melanoma. J Pathol 1998; 186: $51-58$

${ }^{42}$ Massi D, Franchi A, Borgognoni L, Reali UM, Santucci M. Osteonectin expression correlates with clinical outcome in thin cutaneous malignant melanomas. Hum Pathol 1999; 30: 339- 344

\section{Buchbesprechung}

\section{Medizin im Nationalsozialismus am Beispiel der Dermatologie}

Elsner, P., U. Zwiener (Hrsg.)

Heft 25, 88 Seiten. Palm \& Enke, Collegium Europaeum Jenense, Jena, Erlangen 2002. 5,50 €. ISBN 3-7896-0663-4

Wir leben in einem Zeitalter stürmischer wissenschaftlicher Entwicklungen und der Extreme, das gilt insbesondere für einige Fachbereiche der Medizin und Biologie. Trotz aller Erfolge und Neuerungen auf diesen Gebieten erweist es sich dabei als notwendig, von Zeit zu Zeit aus wissenschaftshistorischer Sicht eine Standortbestimmung vorzunehmen, sich der Geschichte dieser Fortschritte bewusst zu werden, um so aus der Historiographie neue Kraft und Impulse für die bevorstehenden Aufgaben zu gewinnen. Mit den politischen Umwälzungen in Deutschland zu Beginn der 1990er Jahre bot sich sowohl den Fachwissenschaftlern als auch den Wissenschaftshistorikern die einmalige Möglichkeit, bis dahin gefärbte oder gar verfälschende Überlieferungen zur Geschichte ihrer Fachdisziplinen, wie besonders der Medizin etc. - sowohl in West- als auch in Ostdeutschland - grundsätzlich neu zu überdenken, die darin vorgebrachten Argumente zu verifizieren und damit einer objektiven Analyse zu unterziehen. Ein teilweise sehr konträres Problemfeld offenbarte sich besonders in der bis dahin nur ungenügend erfolgten wissenschaftlichen Aufarbeitung des Wechselverhältnisses von Wissenschaft, Politik und Ideologie während der Zeit des Dritten Reiches. Hier rückten hauptsächlich verschiedene Fachdisziplinen, wie besonders der Medizin, brisante wissenschaftspolitische Themengebiete, Fakultäts- und Institutsgeschichten, auch einzelne Protagonisten der Medizin sowie der Biowissenschaften usw. ins Blickfeld der Analyse. Der vorliegende Sammelband, ediert vom Dermatologen Peter Elsner und dem Pathophysiologen Ulrich Zwiener (beide Jena), schließt unmittelbar daran an und wirft - exemplarisch am Beispiel der Dermatologie aufgezeigt - Licht und Schatten auf ein Kapitel deutscher Medizingeschichte. Es ist den beiden Herausgebern zu danken, sich mit einer vorausgehenden öffentlichen Vortragsreihe von 2000 bis 2001 und der Publikation eines Sammelbandes dieser speziellen Thematik angenommen zu haben. Sie ergänzen dabei nachhaltig auch die Arbeit der seit 2000 an der Friedrich-Schiller-Universität Jena bestehenden Senatskommission zur Aufarbeitung der Jenaer Universitätsgeschichte im 20. Jahrhundert. Nach einer Einführung mit Problemaufriss seitens der Herausgeber folgen die Fachbeiträge. Hier skizziert Susanne Zimmermann eingangs in dem Beitrag über „Fakten und Legenden“ ausgewählte Beispiele der Geschichte der Medizinischen Fakultät der Universität Jena zwischen 1933 und 1945 (S.12-34). Aufgrund neuer Archivalien erfährt der Leser so beispielsweise Neues und Wissenswertes über Handlungen, Hal- tungen und so genannte ,Verstrickungen ““ (S.12) des Internisten Heinrich W. Veil und des Dermatologen Josef Hämel. Daran schließen sich Albrecht Scholz Ausführungen über „Aufstieg und Ende der jüdischen Dermatologen in Deutschland“ an (S. 34-52). Bei dieser Analyse, die u. a. auch die Aufklärung von Lebenswegen jüdischer Dermatologen zum Inhalt hat, stützt sich der Autor auf Ergebnisse seiner umfassenden Studien zur Geschichte der Dermatologie in Deutschland von 1999. Im Anschluss an Scholz thematisiert Wolfgang Weyers mit der Dermatologie im Dritten Reich den „Niedergang eines Fachgebietes“ und belegt dabei die Verstrickung vieler Dermatologen wie beispielsweise Bodo Spiethoff, Martin Schubert, Julius Dörffel und Ernst Heinrich Brill mit der nationalsozialistischen Wissenschaftspolitik (S.53-66). Die führende Stellung der deutschen Dermatologie in der Welt durch die Leistungen aus der ersten Hälfte des 20. Jahrhunderts, gerade durch die jüdischen Dermatologen wesentlich erbracht, wurde nachhaltig zerstört. Es ist für jeden aufmerksamen Leser erschütternd, mit welcher brutalen Konsequenz die jüdischen Ärzte um ihre beruflichen Möglichkeiten gebracht wurden. Jene, die nicht emigrierten, kamen schließlich fast alle um. Das Gleiche traf für die jüdischen Patienten zu, die von „Ariern“ nicht mehr behandelt werden durften. Peter Elsner beschließt mit seinen Bemerkungen zum „Dichter-Dermatologen Gottfried Benn" den Rahmen der Fachbeiträge (S. 67-78). Der Sammelband wird schließlich abgerundet - an Stelle eines Nachwortes - durch den Abdruck einer Erklärung der Medizinischen Fakultät der Universität Jena vom 18. April 2000 zum Umgang mit den Verbrechen aus der Zeit des Nationalsozialismus, die an dieser Fakultät begangen wurden. Die Fakultät erklärt ihre Betroffenheit darüber, will das Andenken der Opfer bewahren, die Erinnerung an die Ereignisse wachhalten, die Studenten und Mitarbeiter informieren und die Tragweite der Verbrechen deutlich machen, damit solches Geschehen sich nicht wiederholen kann. Es folgen ein erläuterndes Glossar für nichtmedizinische Leser, die Autorenanschriften, sowie eine Übersicht über die bisher in dieser Reihe erschienenen Bände des Collegium Europaeum Jenense. Dem handlichen Buch ist eine weite Verbreitung zu wünschen. Es ist klar, dass mit diesem Sammelband bei weitem nicht alle Fragen zu diesem Thema umfassend beantwortet werden konnten, sondern durch die Lektüre auch zahlreiche neue aufgeworfen werden, denen wir uns stellen müssen. Gerade deshalb weckt es vielleicht bei dem einen oder anderen Leser Interesse, sich mit diesem speziellen Problemfeld - der historischen Analyse von Fachdisziplinengeschichten - innerhalb der Medizin (wieder) zu beschäftigen.

U. Hoßfeld, Jena U. Wollina, Dresden 